Obesity, Food Addiction and Executive Functions -1-

\title{
Food Addiction and Impaired Executive Functions in Women with Obesity
}

Trevor Steward ${ }^{1,2 \#}$, Gemma Mestre-Bach ${ }^{1,2 \#}$, Cristina Vintró-Alcaraz ${ }^{1,2}$, María Lozano-Madrid ${ }^{1,2}$, Zaida Agüera $^{1,2}$, José A. Fernández-Formoso ${ }^{1}$; Roser Granero ${ }^{1,3}$, Susana Jiménez-Murcia ${ }^{1,2,4}$, Nuria Vilarrasa ${ }^{5,6}$; Amador García-Ruiz-de-Gordejuela ${ }^{7}$; Misericordia Veciana de las Heras ${ }^{4}$; Nuria Custal ${ }^{2}$; Nuria Virgili ${ }^{4,5}$, Rafael López-Urdiales ${ }^{5}$, Ashley N. Gearhardt ${ }^{8}$; José M. Menchón ${ }^{2,4,9}$; Carles Soriano-Mas ${ }^{2,3,9}$; Fernando Fernández-Aranda ${ }^{1,2,4^{*}}$

${ }^{l}$ Ciber Fisiopatologia Obesidad y Nutrición (CIBEROBN), Instituto Salud Carlos III, Barcelona, Spain.

${ }^{2}$ Department of Psychiatry, Bellvitge University Hospital -IDIBELL, Barcelona, Spain.

${ }^{3}$ Departament de Psicobiologia i Metodologia de les Ciències de la Salut. Universitat Autònoma de Barcelona, Barcelona, Spain.

${ }^{4}$ Department of Clinical Sciences, School of Medicine, University of Barcelona, Spain

${ }^{5}$ Department of Endocrinology and Nutrition, University Hospital of Bellvitge-IDIBELL, Barcelona, Spain.

${ }^{6}$ CIBERDEM-CIBER de Diabetes y Enfermedades Metabólicas Asociadas, Instituto de Salud Carlos III, Barcelona, Spain.

${ }^{7}$ Bariatric and Metabolic Surgery Unit, Service of General and Gastrointestinal Surgery, University Hospital of BellvitgeIDIBELL, Barcelona, Spain

${ }^{8}$ Department of Psychology, University of Michigan, Ann Arbor, MI, USA.

${ }^{9}$ Ciber Salud Mental (CIBERSAM), Instituto Salud Carlos III, Barcelona, Spain.

\section{*Correspondence:}

This is the author manuscript accepted for publication and has undergone full peer review but has not been through the copyediting, typesetting, pagination and proofreading process, which may lead to differences between this version and the Version of Record. Please cite this article as doi: $10.1002 /$ erv.2636

This article is protected by copyright. All rights reserved. 
Obesity, Food Addiction and Executive Functions -2-

Fernando Fernández-Aranda, Eating Disorders Unit-Department of Psychiatry and CIBERObn, University Hospital of Bellvitge-IDIBELL, c/ Feixa Llarga s/n, 08907-Barcelona, Spain. Tel. +34-932607227. E-mail: ffernandez@bellvitgehospital.cat

\#Shared first authorship

Running title: Obesity, Food Addiction and Execute Functions

\section{Funding:}

This manuscript and research was supported by grants from the Ministerio de Economía y Competitividad (PSI2015-68701-R), Ministerio de Sanidad, Servicios Sociales e Igualdad (PR338/17), Instituto de Salud Carlos III (ISCIII) (FIS PI14/00290 and PI17/01167) and co-funded by FEDER funds /European Regional Development Fund (ERDF), a way to build Europe. CIBERObn and CIBERSAM are both initiatives of ISCIII. The funders had no role in the study design, data collection and analysis, decision to publish, or preparation of the manuscript. This research was supported by a PNSD (PR338/17-MSSSI) grant. GMB is supported by a predoctoral AGAUR grant (2018 FI_B2 00174), co-financed by the European Social Fund, with the support of the Secretaria d'Universitats i Recerca del Departament d'Economia i Coneixement de la Generalitat de Catalunya. MLM and CVA are supported by a predoctoral Grant of the Ministerio de Educación, Cultura y Deporte (FPU15/0291; FPU16/01453). CSM is supported by a 'Miguel Servet' contract from the Carlos III Health Institute (Madrid, Spain) (CPII16/00048).

Conflict of interest: The authors declare no conflict of interest. 


\begin{abstract}
Background: Individuals with obesity often report suffering from addiction-like symptoms. As in addictions, deficits in executive function domains, such as decision-making and sustained attention, are found in obesity. No study to date has examined the associations between food addiction, obesity and neuropsychological performance.
\end{abstract}

Method: 33 adult women with obesity and 36 healthy weight controls completed the Yale Food Addiction Scale Version 2.0, a validated instrument used to assess food-related addictive behaviors. Additionally, participants completed computerized versions of the Iowa Gambling Task (IGT) and Conners' Continuous Performance Test (CPT-II) to examine decision-making and attentional control, respectively.

Results: Food addiction criteria were met in $24.2 \%$ of the participants with obesity and in $2.8 \%$ of the control group. In the obesity group, food addiction severity levels were negatively correlated with overall scores on the IGT. Participants with obesity meeting criteria for food addiction committed more omissions and perseveration errors on the CPT-II compared to those without food addiction.

Conclusions: Our results point to an association between food addiction severity levels and impairments in decision-making and attentional capacity in individuals with obesity. Given the heterogeneity found in obesity, it stands to reason that this subset of patients with food addiction could potentially benefit from interventions targeting neuropsychological deficits.

\title{
HIGHLIGHTS
}

1) Food addiction and neuropsychological functioning were assessed in a sample of women with obesity and healthy weight controls.

2) Higher food addiction severity was positively associated with poorer decision-making on the Iowa Gambling Task.

This article is protected by copyright. All rights reserved. 
3) Women in the obese group with food addiction presented greater attentional deficits than women without food addiction.

\section{KEYWORDS}

Obesity, food addiction, executive functions, decision-making, attention.

\section{INTRODUCTION}

Food addiction, a developing construct that posits that the overconsumption of highly palatable food is sustained by addictive processes, has received increased interest from clinicians and researchers alike (Gearhardt, Corbin, \& Brownell, 2009; Novelle \& Diéguez, 2018; Nunes-Neto et al., 2018; Treasure, Leslie, Chami, \& Fernández-Aranda, 2018). Individuals with obesity and/or eating disorders often report addiction-like symptoms, such as preoccupation with obtaining a desired food, high impulsivity levels, functional impairment, the excessive intake of unhealthy foods despite adverse health consequences, tolerance and withdrawal (Morris, Beilharz, Maniam, Reichelt, \& Westbrook, 2015; Schulte, Yokum, Potenza, \& Gearhardt, 2016). In addition to the symptomatological similarities between excessive food intake and substance abuse, recent evidence suggests that the neural reward centers are activated by highly-palatable foods in a manner akin to drugs of abuse (Leigh \& Morris, 2018; Morin et al., 2017; Schulte, Avena, \& Gearhardt, 2015).

The Yale Food Addiction Scale Version 2.0 (YFAS 2.0), which uses adapted DSM-5 Substance-Related and Addictive Disorders criteria (APA, 2013; Meule \& Gearhardt, 2014), is the most commonly used instrument to assess food-related addictive behaviors. The prevalence of food addiction in people with excess weight and obesity has been inconsistent, with prevalence rates ranging between $7 \%$ and $56 \%$ (Ceccarini, Manzoni, Castelnuovo, \& Molinari, 2015; Meule, Heckel, Jurowich, Vögele, \& Kübler, 2014; Pursey, Stanwell, Gearhardt, Collins, \& Burrows, 2014). This mismatch between results could be 
Obesity, Food Addiction and Executive Functions -5explained bearing in mind the heterogeneity found in people with obesity and the presence of eating disorder comorbidity (de Vries \& Meule, 2016; Granero et al., 2014). Moreover, it has been postulated that food addiction severity might serve as an indicator of subclinical binge eating behaviors (Alvarenga et al., 2014; Cope \& Gould, 2017; Davis, 2017), overall eating disorder severity (Albayrak et al., 2017; Granero et al., 2014), and dysfunctional personality traits (Granero et al., 2018; Jiménez-Murcia et al., 2017; Wolz, Granero, \& Fernández-Aranda, 2017).

Relatedly, obesity is linked to deficits in a variety of executive function domains such as decision making, response inhibition and cognitive flexibility (Siervo et al., 2011; Smith, Hay, Campbell, \& Trollor, 2011; Stinson, Krakoff, \& Gluck, 2018; Yang, Shields, Guo, \& Liu, 2018). For example, numerous studies have demonstrated using the Iowa Gambling Task (IGT) that adults with obesity present significant deficits in decision-making (Brogan, Hevey, \& Pignatti, 2010; Danner, Ouwehand, Van Haastert, Hornsveld, \& De Ridder, 2012; Fagundo et al., 2016; Fagundo et al., 2012; Pignatti et al., 2006; Rotge, Poitou, Fossati, Aron-Wisnewsky, \& Oppert, 2017). This tendency suggests that the excess intake of highly palatable foods might be underpinned by an inability to successfully weigh the risks of reward and punishment (Fagundo et al., 2012). Furthermore, researchers have measured deficits in inhibitory control amongst individuals with substance abuse through tasks such as the Go/NoGo Task and the Stop-Signal Task. In corroboration with these results, neuroimaging studies revealed consistent irregularities in prefrontal brain function amongst individuals with substance abuse, thus indicating defective executive functioning in this population (Luijten et al., 2014; Smith, Mattick, Jamadar, \& Iredale, 2014). Despite the evident parallels between decision-making impairments and obesity, no studies to date have taken into account the role that food addiction could play in this relationship. 
Obesity, Food Addiction and Executive Functions -6Some researchers have proposed that obesity and attention deficit/hyperactivity-disorder (ADHD) might share a common neurocognitive phenotype, characterized by deficits in hot executive functions, associated with impaired inhibitory control and impulsivity have (Lavagnino, Arnone, Cao, Soares, \& Selvaraj, 2016; Preuss, Pinnow, Schnicker, \& Legenbauer, 2017). Previous studies have observed that people with obesity performed significantly worse on tasks assessing domains such as interference control, memory span length, and reward sensitivity in comparison to healthy weight controls (Aiello et al., 2018; Fagundo et al., 2012; Farooq, Gibson, Reilly, \& Gaoua, 2018; Song et al., 2016). Specifically, interference control tasks assess the capacity of suppressing inappropriate actions that could interfere with achieving a long-term goal and evidence suggests that some individuals with obesity display difficulty inhibiting automatic or model-free behavior (MacLeod, 1991; Preuss et al., 2017). Lastly, there is increasing evidence to support that obesity has a detrimental effect on overall attentional capacity (Cook et al., 2017; Fagundo et al., 2012; Siervo et al., 2011; Tsai, Huang, \& Tsai, 2017). Even though these neuropsychological alterations have been widely explored in obesity, to the best of our knowledge, no study has examined the association between food addiction and these deficits in people with obesity.

\subsection{Aims \& Hypotheses}

In the present study, we aimed to assess the prevalence and severity level of food addiction in a sample of women with obesity (OB) and healthy controls (HC), and to explore the associations between neuropsychological performance, impulsivity traits, and food addiction. Specifically, we sought to compare neuropsychological functioning in individuals with and without food addiction and to examine the associations between food addiction severity, impulsivity, and executive functioning.

\section{METHODS}

\subsection{Sample}


Obesity, Food Addiction and Executive Functions -7-

Our sample consisted of 33 women with $\mathrm{OB}$ who were recruited from the Bariatric and Metabolic Surgery Unit, and the Endocrinology and Nutrition Unit at Bellvitge University Hospital (Barcelona, Spain). These patients were compared to 36 healthy-weight controls (HC) that were recruited from the same University Hospital setting. As part of the screening procedure for bariatric surgery, all patients with OB were screened for the presence of an eating disorder via a semi-structured interview, which took place at the Eating Disorders Unit of Bellvitge University Hospital. Patients who received an eating disorder diagnosis were referred to treatment and not included in the study sample. All participants underwent The Mini-International Neuropsychiatric Interview (M.I.N.I.) (Sheehan, 1998) with staff psychologists to screen for the presence of a possible psychiatric disorder.

Study inclusion criteria were the following: being female and being between the age of 18 and 55. The study exclusion criteria were: being male, the presence of an organic mental disorder and an intellectual disability, and a history of an eating disorder or any other psychiatric condition. Prior to assessment, HC participants were asked regarding lifetime minimum and maximum BMI, and those who reported having obesity (BMI>30) were excluded from the study sample.

The present study was carried out in accordance with the latest version of the Declaration of Helsinki. The Bellvitge University Hospital Clinical Research Ethics Committee approved the study, and signed informed consent was obtained from all participants.

\subsection{Neuropsychological Tasks and Clinical Measures}

Following the face-to-face interview, participants completed the study questionnaires and neuropsychological tasks whilst accompanied by a staff psychologist.

\section{Anthropometric Measures}

A Tanita BC-420MA was utilized to assess body composition and to calculate BMI. This noninvasive and validated device (Tanita BC- 420MA, Tanita Corp. Tokyo, Japan) uses bioelectrical impedance analysis 
Obesity, Food Addiction and Executive Functions -8to measure weight and body composition variables (Browning et al., 2011). Height was measured via a stadiometer.

Conners' Continuous Performance Test, 2nd edition (CPT-II) (Conners, 2004)

The CPT-II is a computer-based task that involves participants pressing the space bar in response to visual stimuli (i.e. letters on computer screen) that are presented over a span of 14-min. The CPT-II provides information about the participants' omission and commission error rates, reaction time, and response variability, which represent an assessment of sustained attention and inhibitory control. Higher scores on the CPT-II indicate worse performance.

Iowa Gambling Task (IGT) (Bechara, Damasio, Tranel, \& Damasio, 1997)

On the computerized version IGT used in this study to assess decision-making capacity, subject select 100 cards, over five blocks (20 in each block), from four decks (A, B, C and D). A response is provided indicating either a gain or loss of money after selecting a card. Two decks (A and B) are not advantageous since the final loss of money is greater than the final gain. Decks C and D, however, are advantageous as they provide small, overall gains. Participants are instructed that the final goal of the task is to accumulate as much money as possible. In order to determine the net score for each block, along with the overall net score, the number of cards selected from decks A and B is subtracted from the number of cards selected from decks $\mathrm{C}$ and $\mathrm{D}$ [i.e. $(\mathrm{C}+\mathrm{D}) \mathrm{A}+\mathrm{B})$ ]. Higher scores point to better performance while negative scores point to persistently choosing disadvantageous decks.

Psychometric measures

Yale Food Addiction Scale Version 2.0 (YFAS 2.0) (Gearhardt, Corbin, \& Brownell, 2016)

This self-report questionnaire consists of 35 items scored on an eight-level Likert scale (from $0=$ never to 7=every day) and adapted to assess addictive eating behaviors based on DSM-5 substance-related and addictive disorders criteria (APA, 2013). 
Obesity, Food Addiction and Executive Functions -9-

The Spanish YFAS 2.0 is has been validated in a Spanish-speaking population and the scoring guidelines used in the original validation of the YFAS 2.0 were followed for this study (Granero et al., 2018). These scoring guidelines produces two measurements: (a) a continuous symptom count score that reflects the number of fulfilled diagnostic criteria (ranging from 0 to 11); and (b) a food addiction threshold based on the number of symptoms (at least 2) and self-reported clinically significant impairment or distress. This final measurement allows for the binary classification of food addiction (present versus absent). Based on the revised DSM-5 taxonomy, the YFAS 2.0 also provides severity cutoffs: mild (2-3 symptoms), moderate (4-5 symptoms), and severe (6-11 symptoms). The Spanish version of the YFAS 2.0 has excellent accuracy in discriminating between $\mathrm{HC}$ and eating-disorder subsamples $(\kappa=0.75)$ and its internal reliability coefficient is excellent ( $\alpha=0.94)$ (Granero et al., 2018).

Impulsive Behavior Scale (UPPS-P) (Whiteside, Lynam, Miller, \& Reynolds, 2001)

The UPPS-P measures five facets of impulsive behavior through self-report. It categorizes impulsivity into five independent dimensions: sensation seeking, which refers to one's disposition to seek exciting experiences; (lack of) perseverance, which reflects the tendency to not persist in an activity that can be arduous; (lack of) premeditation shows the tendency to act without considering the consequences of behavior; and positive and negative urgency refer to emotionally charged impulsive behaviors in response to positive or negative moods. The Spanish-language adaptation of the UPPS-P showed good reliability (Cronbach's $\alpha$ between 0.79 and 0.93) and external validity (Verdejo-García, Lozano, Moya, Alcázar, \& Pérez-García, 2010).

Eating Disorders Inventory-2 (Garner, 1991)

The Eating Disorders Inventory-2 (EDI-2) is a 91-item self-report questionnaire that assesses the following eating disorder factors: drive for thinness, bulimia, body dissatisfaction, ineffectiveness, perfectionism, interpersonal distrust, interoceptive awareness, maturity fears, asceticism, impulse 
Obesity, Food Addiction and Executive Functions -10-

regulation and social insecurity (Garner, 1991). A validated Spanish version of this questionnaire was used for this study (Garner, 1998) and it has an excellent internal consistency $(\alpha=.964)$.

\subsection{Statistical analyses}

Statistical analysis was carried out with Stata15 for Windows. First, the comparison of the prevalence and severity of food addiction between the two study groups (HC versus OB) was based on chi-square $\left(\chi^{2}\right)$ tests. Comparisons between groups based on food addiction severity (defined as the total number of fulfilled YFAS 2.0 criteria) was based on analysis of variance (ANOVA) procedures. Next, the associations between food addiction scores and study measures (neuropsychological performance, impulsivity levels and eating disorder severity) were estimated through partial correlation coefficients. Finally, comparisons of $\mathrm{OB}$ patients with and without food addiction were carried out using ANOVA procedures.

In this study, all the analysis involving executive function measures were controlled for the covariates age and the years of education, while the analyses of other clinical variables (impulsivity and ED severity) were adjusted for age.

Effect size was measured through Cohen's- $d$ coefficient for mean and proportional comparisons $(|d|>0.2$ was considered low effect size, $|d|>0.5$ was considered moderate and $|d|>0.8$ was considered large; (Kelley \& Preacher, 2012). For partial correlations, and due to the strong association between this coefficient and sample size, practical relevance was based coefficient measures $(|r|>0.10$ was considered low effect size, $|r|>0.24$ was considered medium effect size and $|r|>0.37$ was considered large effect size; (Rosnow \& Rosenthal, 1996).

Finner's method was used to control Type-I error due to multiple statistical comparisons. This procedure, included in Family-wise error rate stepwise procedures, is based on a step-down approach using a slightly 
Obesity, Food Addiction and Executive Functions -11adapted Sidak-method, and it has been demonstrated to provide more powerful results than Bonferroni correction (Finner, 1993).

\section{RESULTS}

\subsection{Sample characteristics}

No differences between groups were found regarding years of education (HC: mean=15.3 years, $\mathrm{SD}=1.8$; OB: mean=14.8 years, $\left.\mathrm{SD}=2.1 ; F_{d f=1 / 67}=1.09, p=.299\right)$. Mean age for the HC group was 30.6 years $(\mathrm{SD}=12.6)$, and 38.7 years for the $\mathrm{OB}$ group $(\mathrm{SD}=10.3)\left(F_{d f=1 / 67}=8.28, p=.005\right)$. In terms of BMI, as expected, means statistically differed between groups $\left(\mathrm{HC}\right.$ : mean $=21.3 \mathrm{~kg} / \mathrm{m}^{2}, \mathrm{SD}=2.1$; $\mathrm{OB}$ : mean $=42.4$ $\left.\mathrm{kg} / \mathrm{m}^{2}, \mathrm{SD}=6.9 ; F_{d f=1 / 67}=30.5, p=<.001\right)$.

Table S1 (supplementary material) includes the distribution of all the measures of the study, as well as the consistency (Cronbach's alpha) obtained in the study sample.

\subsection{Food addiction prevalence and comparisons between groups}

Table 1 includes the prevalence of the presence/absence of food addiction and food addiction severity levels. Comparisons between groups can also be found in Figure 1. Food addiction criteria were met in $24.2 \%$ of the participants with $\mathrm{OB}$ and in $2.8 \%$ of the HC group $(p=.008 ; d=0.66)$. Regarding food addiction severity, $9.1 \%$ of the OB patients had moderate food addiction severity and $15.2 \%$ high severity, while the $2.8 \%$ of $\mathrm{HC}$ group had high food addiction severity levels.

--- Insert Table 1 ---

--- Insert Figure 1 ---

The last row of Table 1 includes a comparison of the means of met food addiction criteria in each group (see the second panel of Figure 1). Significant differences between groups emerged with the OB group endorsing an average of 2.6 food addiction criteria versus 0.7 for the $\mathrm{HC}$ group $(p=.001, d=0.87)$.

\subsection{Associations between food addiction and neuropsychological measures}


Obesity, Food Addiction and Executive Functions -12-

Table 2 includes partial correlations estimating the association between the fulfilled food addiction criteria with the study neuropsychological and clinical measures (performance on the IGT and CPT tasks, impulsivity as measured by the UPPS-P, and ED severity as measured by the EDI-2 total score). In the HC group, positive associations were found between the number of food addiction criteria with impulsivity dimensions and ED severity. In the OB group, food addiction severity levels were negatively correlated with performance on the IGT and positively correlated with negative urgency.

--- Insert Table 2 ---

Table 3 includes comparisons between patients in the OB group with and without food addiction. Patients meeting criteria for food addiction obtained higher means in the number of omissions and perseveration errors on the CPT-II compared to patients in the OB group without food addiction. These patients also endorsed higher levels of negative urgency and overall ED severity. Lower mean scores on the first block of the IGT were also found in the OB group meeting criteria for food addiction.

\section{DISCUSSION}

The purpose of this study was to examine the interplay between food addiction severity and neuropsychological functioning in two specific domains (decision making and attentional capacity) in a sample of women with $\mathrm{OB}$ and $\mathrm{HC}$. We also sought to differentiate neuropsychological profiles in the group with OB by distinguishing between patients with and without food addiction.

Regarding food addiction prevalence, $24.2 \%$ of the participants with OB met criteria for food addiction, whereas prevalence in the $\mathrm{HC}$ group was $2.8 \%$. In the case of $\mathrm{OB}$, food addiction prevalence levels were within the range of previous studies using the YFAS 2.0 in samples with obesity, ranging from $17.2 \%$ to 19.3\% (Hauck, Weiß, Schulte, Meule, \& Ellrott, 2017; Schulte \& Gearhardt, 2018). Furthermore, the 
Obesity, Food Addiction and Executive Functions -13-

prevalence rate in our control sample is similar to the values reported in previous studies using adult (Granero et al., 2018) and adolescent samples (Mies et al., 2017).

In terms of decision-making, our IGT results indicate that increased food addiction severity in the OB group was positively associated with poorer overall performance, suggesting that a deficit in making adaptive decisions could be underpinned by addictive symptomatology in some individuals with obesity. Patients with binge eating disorder and OB alike often report suffering from a lack of behavioral control during recurrent overeating episode and thus fail to adjust behavior in the face of negative consequences (Fagundo et al., 2012; Reiter, Heinze, Schlagenhauf, \& Deserno, 2017). Other studies have identified a link between decision-making impairment and binge eating severity (Danner et al., 2012), and it is plausible that similar impairments are present in sub-threshold binge eating disorder patients who present food addiction symptoms. In line with our UPPS-P findings, numerous studies have indicated that individuals with food addiction experience difficulty controlling impulses when experiencing a negative mood i.e. negative urgency (Rose, Nadler, \& Mackey, 2017; Wolz et al., 2016, 2017), and future studies should examine how decision-making is influenced in "hot" and "cold" executive function settings (Appelhans, French, Pagoto, \& Sherwood, 2016).

Our results also point to an association between food addiction severity levels and diminished attentional capacity in individuals with obesity. Specifically, patients with obesity meeting food addiction criteria made significantly more omissions on the CPT than those without food addiction. A greater number of omissions is indicative of a participant not responding to the target stimuli during presentation, generally due to difficulties with focusing one's attention (Conners, 2004). Obesity has been linked to impaired attentional capacity and our findings suggest that individuals with food addiction symptomatology may be at greater risk of presenting attentional deficits (Cook et al., 2017; Fagundo et al., 2012; Siervo et al., 
Obesity, Food Addiction and Executive Functions -142011; Tsai et al., 2017). A recent meta-analysis identified a moderate overall association between attention-deficit/hyperactivity disorder (ADHD) and being overweight/obesity in adults (Nigg et al., 2016) and it would be of interest to examine whether obese individuals with food addiction have a greater likelihood of having ADHD than those without.

\section{Limitations}

This study is not without its limitations. First, our study design is cross-sectional in nature and claims regarding causality cannot be made. Future longitudinal studies should examine the extent to which cognitive function improves after weight loss in patients with food addiction (Veronese et al., 2017). Second, the size of our sample was modest [power calculation for the main analysis of the study, the estimation of the correlation between food addiction with neuropsychological measures (considering the parameters $\alpha=0.05, \rho=0.37$ and sample size $n=36$-equal to the sample of the clinical group-) resulted in $1-\beta=0.61]$ and confirmatory studies with larger samples are required to confirm the associations identified between food addiction severity and executive functions. Lastly, our sample with obesity was largely made up of middle-aged adults and it would be of clinical interest to explore whether similar impairments in neuropsychological functioning are present in younger samples with food addiction (Nigg et al., 2016).

\section{Conclusion and clinical implications}

The findings of this study provide an initial indication of the associations between food addiction severity and impairments in decision-making and attentional capacity in individuals with obesity. Obesity is a heterogeneous condition and it could be postulated that patients with food addiction could stand to benefit from interventions targeting these identified neuropsychological deficits. For example, interventions

utilizing attentional bias modification to change evaluations of substance-related cues have thus far 
Obesity, Food Addiction and Executive Functions -15provided mixed results, and it would be of interest to examine whether patients with food addiction respond differently to attentional bias modification than those without (Field et al., 2016). Highly prevalent cognitive behavior therapy approaches for overeating currently do not favor avoidance or abstinence in the same way as addiction treatments (Wilson, 2010). Future studies should test whether individuals with food addiction present improved treatment outcomes to approaches that advocate the controlled consumption of specific foods in comparison to other treatments (Ziauddeen, Alonso-Alonso, Hill, Kelley, \& Khan, 2015). 


\section{REFERENCES}

Aiello, M., Ambron, E., Situlin, R., Foroni, F., Biolo, G., \& Rumiati, R. I. (2018). Body weight and its association with impulsivity in middle and old age individuals. Brain and Cognition, 123, 103-109.

Albayrak, Ö., Föcker, M., Kliewer, J., Esber, S., Peters, T., de Zwaan, M., \& Hebebrand, J. (2017). Eating-related Psychopathology and Food Addiction in Adolescent Psychiatric Inpatients. European Eating Disorders Review, 25(3), 214-220.

Alvarenga, M. S., Koritar, P., Pisciolaro, F., Mancini, M., Cordás, T. A., \& Scagliusi, F. B. (2014). Eating attitudes of anorexia nervosa, bulimia nervosa, binge eating disorder and obesity without eating disorder female patients: Differences and similarities. Physiology and Behavior, 131, 99-104.

American Psychiatric Association. (2013). Diagnostic and Statistical Manual of Mental Disorders, Fifth Edition. Washington, DC: American Psychiatric Association.

Appelhans, B. M., French, S. A., Pagoto, S. L., \& Sherwood, N. E. (2016). Managing temptation in obesity treatment: A neurobehavioral model of intervention strategies. Appetite, 96, 268-279.

Bechara, A., Damasio, H., Tranel, D., \& Damasio, a R. (1997). Deciding advantageously before knowing the advantageous strategy. Science (New York, N.Y.), 275(5304), 1293-1295.

Brogan, A., Hevey, D., \& Pignatti, R. (2010). Anorexia, bulimia, and obesity: Shared decision making deficits on the Iowa Gambling Task (IGT). Journal of the International Neuropsychological Society, 16(4), 711-715.

Browning, L. M., Mugridge, O., Dixon, A. K., Aitken, S. W., Prentice, A. M., \& Jebb, S. A. (2011). Measuring abdominal adipose tissue: Comparison of simpler methods with MRI. Obesity Facts, $4(1), 9-15$. 
Obesity, Food Addiction and Executive Functions -17-

Ceccarini, M., Manzoni, G. M., Castelnuovo, G., \& Molinari, E. (2015). An Evaluation of the Italian Version of the Yale Food Addiction Scale in Obese Adult Inpatients Engaged in a 1-Month-WeightLoss Treatment. Journal of Medicinal Food, 18(11), 1281-1287.

Conners, C. (2004). Conners' continuous performance test (CPT II) version 5 for windows: Technical guide and software manual. Toronto, Canada: Multi-Health Systems.

Cook, R. L., O’Dwyer, N. J., Donges, C. E., Parker, H. M., Cheng, H. L., Steinbeck, K. S., ... O’Connor, H. T. (2017). Relationship between Obesity and Cognitive Function in Young Women: The Food, Mood and Mind Study. Journal of Obesity, 2017, 1-11.

Cope, E. C., \& Gould, E. (2017). New Evidence Linking Obesity and Food Addiction. Biological Psychiatry, 81(9), 734-736.

Danner, U. N., Ouwehand, C., Van Haastert, N. L., Hornsveld, H., \& De Ridder, D. T. D. (2012). Decision-making impairments in women with binge eating disorder in comparison with obese and normal weight women. European Eating Disorders Review, 20(1), e56-62.

Davis, C. (2017). A commentary on the associations among 'food addiction', binge eating disorder, and obesity: Overlapping conditions with idiosyncratic clinical features. Appetite, 115, 3-8.

de Vries, S. K., \& Meule, A. (2016). Food Addiction and Bulimia Nervosa: New Data Based on the Yale Food Addiction Scale 2.0. European Eating Disorders Review, 24(6), 518-522.

Fagundo, A. B., de la Torre, R., Jiménez-Murcia, S., Agüera, Z., Granero, R., Tárrega, S., ... FernándezAranda, F. (2012). Executive functions profile in extreme eating/weight conditions: From Anorexia Nervosa to Obesity. PLoS ONE, 7(8).

Fagundo, A. B., Jiménez-Murcia, S., Giner-Bartolomé, C., Agüera, Z., Sauchelli, S., Pardo, M., ... 
Obesity, Food Addiction and Executive Functions -18-

Fernández-Aranda, F. (2016). Modulation of irisin and physical activity on executive functions in obesity and morbid obesity. Scientific Reports, 6, 30820.

Farooq, A., Gibson, A.-M., J Reilly, J., \& Gaoua, N. (2018). The Association between Obesity and Cognitive Function in Otherwise Healthy Premenopausal Arab Women. Journal of Obesity, 2018, 1741962.

Field, M., Werthmann, J., Franken, I., Hofmann, W., Hogarth, L., \& Roefs, A. (2016). The role of attentional bias in obesity and addiction. Health Psychology, 35(8), 767.

Finner, H. (1993). On a Monotonicity Problem in Step-Down Multiple Test Procedures. Journal of the American Statistical Association, 88(423), 920-923.

Garner, D. M. (1991). Eating Disorder Inventory-2. Book, Odessa: Psychological Assessment Resources.

Garner, D. M. (1998). Inventario de Trastornos de la Conducta Alimentaria (EDI-2)-Manual. Madrid: TEA.

Gearhardt, A. N., Corbin, W. R., \& Brownell, K. D. (2009). Food Addiction. Journal of Addiction Medicine, 3(1), 1-7.

Gearhardt, A. N., Corbin, W. R., \& Brownell, K. D. (2016). Development of the Yale Food Addiction Scale Version 2.0. Psychology of Addictive Behaviors, 30(1), 113-121.

Granero, R., Hilker, I., Agüera, Z., Jiménez-Murcia, S., Sauchelli, S., Islam, M. A., ... Fernández-Aranda, F. (2014). Food addiction in a Spanish sample of eating disorders: DSM-5 diagnostic subtype differentiation and validation data. European Eating Disorders Review, 22(6), 389-396.

Granero, R., Jiménez-Murcia, S., Gearhardt, A., Agüera, Z., Aymamí, N., Gómez-Peña, M., ... Fernández-Aranda, F. (2018). Clinical Correlates and Validation of the Spanish version of the Yale 
Obesity, Food Addiction and Executive Functions -19Food Addiction Scale 2.0 (YFAS 2.0) and in a Sample of Eating Disorder, Gambling Disorder and Healthy Control Participants. Frontiers in Psychiatry, 9, 208.

Hauck, C., Weiß, A., Schulte, E. M., Meule, A., \& Ellrott, T. (2017). Prevalence of "Food Addiction" as Measured with the Yale Food Addiction Scale 2.0 in a Representative German Sample and Its Association with Sex, Age and Weight Categories. Obesity Facts, 10(1), 12-24.

Jiménez-Murcia, S., Granero, R., Wolz, I., Baño, M., Mestre-Bach, G., Steward, T., ... FernándezAranda, F. (2017). Food Addiction in Gambling Disorder: Frequency and Clinical Outcomes. Frontiers in Psychology, 8, 473.

Kelley, K., \& Preacher, K. (2012). On effect size. Psychological Methods, 17(2), 137-152.

Lavagnino, L., Arnone, D., Cao, B., Soares, J. C., \& Selvaraj, S. (2016). Inhibitory control in obesity and binge eating disorder: A systematic review and meta-analysis of neurocognitive and neuroimaging studies. Neuroscience \& Biobehavioral Reviews, 68, 714-726.

Leigh, S. J., \& Morris, M. J. (2018). The role of reward circuitry and food addiction in the obesity epidemic: An update. Biological Psychology, 131, 31-42.

Luijten, M., Machielsen, M., Veltman, D., Hester, R., de Haan, L., \& Franken, I. (2014). Systematic review of ERP and fMRI studies investigating inhibitory control and error processing in people. Journal of Psychiatry \& Neuroscience, 39(3), 149-169.

MacLeod, C. M. (1991). Half a century of research on the Stroop effect: an integrative review. Psychological Bulletin, 109(2), 163-203.

Mallorquí-Bagué, N., Fagundo, A. B., Jimenez-Murcia, S., De La Torre, R., Baños, R. M., Botella, C., ... Fernández-Aranda, F. (2016). Decision making impairment: A shared vulnerability in obesity, 
Obesity, Food Addiction and Executive Functions -20gambling disorder and substance use disorders? PLoS ONE, 11(9).

Meule, A., \& Gearhardt, A. (2014). Food Addiction in the Light of DSM-5. Nutrients, 6(9), 3653-3671.

Meule, A., Heckel, D., Jurowich, C. F., Vögele, C., \& Kübler, A. (2014). Correlates of food addiction in obese individuals seeking bariatric surgery. Clinical Obesity, 4(4), 228-236.

Mies, G. W., Treur, J. L., Larsen, J. K., Halberstadt, J., Pasman, J. A., \& Vink, J. M. (2017). The prevalence of food addiction in a large sample of adolescents and its association with addictive substances. Appetite, 118, 97-105.

Morin, J.-P., Rodríguez-Durán, L. F., Guzmán-Ramos, K., Perez-Cruz, C., Ferreira, G., Diaz-Cintra, S., \& Pacheco-López, G. (2017). Palatable Hyper-Caloric Foods Impact on Neuronal Plasticity. Frontiers in Behavioral Neuroscience, 11, 19.

Morris, M. J., Beilharz, J. E., Maniam, J., Reichelt, A. C., \& Westbrook, R. F. (2015). Why is obesity such a problem in the 21 st century? The intersection of palatable food, cues and reward pathways, stress, and cognition. Neuroscience and Biobehavioral Reviews, 58, 36-45.

Nigg, J. T., Johnstone, J. M., Musser, E. D., Long, H. G., Willoughby, M. T., \& Shannon, J. (2016). Attention-deficit/hyperactivity disorder (ADHD) and being overweight/obesity: New data and metaanalysis. Clinical Psychology Review, 43, 67-79.

Novelle, M. G., \& Diéguez, C. (2018). Food Addiction and Binge Eating: Lessons Learned from Animal Models. Nutrients, 10(1), 71.

Nunes-Neto, P. R., Köhler, C. A., Schuch, F. B., Solmi, M., Quevedo, J., Maes, M., ... Carvalho, A. F. (2018). Food addiction: Prevalence, psychopathological correlates and associations with quality of life in a large sample. Journal of Psychiatric Research, 96, 145-152. 
Obesity, Food Addiction and Executive Functions -21-

Pignatti, R., Bertella, L., Albani, G., Mauro, A., Molinari, E., \& Semenza, C. (2006). Decision-making in obesity: a study using the Gambling Task. Eating and Weight Disorders , 7:1E3W,LD26-132.

Preuss, H., Pinnow, M., Schnicker, K., \& Legenbauer, T. (2017). Improving Inhibitory Control Abilities (ImpulsE)—A Promising Approach to Treat Impulsive Eating? European Eating Disorders Review, 25(6), 533-543.

Pursey, K. M., Stanwell, P., Gearhardt, A. N., Collins, C. E., \& Burrows, T. L. (2014). The prevalence of food addiction as assessed by the yale food addiction scale: A systematic review. Nutrients, 6(10), 4552-4590.

Reiter, A. M. F., Heinze, H.-J., Schlagenhauf, F., \& Deserno, L. (2017). Impaired Flexible Reward-Based Decision-Making in Binge Eating Disorder: Evidence from Computational Modeling and Functional Neuroimaging. Neuropsychopharmacology, 42(3), 628-637.

Rose, M. H., Nadler, E. P., \& Mackey, E. R. (2017). Impulse Control in Negative Mood States, Emotional Eating, and Food Addiction are Associated with Lower Quality of Life in Adolescents with Severe Obesity. Journal of Pediatric Psychology, 1-9.

Rosnow, R., \& Rosenthal, R. (1996). Computing contrasts, effect sizes, and counternulls on other people's published data: General procedures for research consumers. Psychological Methods, 17(2), $137-152$.

Rotge, J.-Y., Poitou, C., Fossati, P., Aron-Wisnewsky, J., \& Oppert, J.-M. (2017). Decision-making in obesity without eating disorders: a systematic review and meta-analysis of Iowa gambling task performances. Obesity Reviews, 18(8), 936-942.

Schulte, E. M., Avena, N. M., \& Gearhardt, A. N. (2015). Which foods may be addictive? The roles of processing, fat content, and glycemic load. PLOS ONE, 10(2). 
Obesity, Food Addiction and Executive Functions -22-

Schulte, E. M., \& Gearhardt, A. N. (2018). Associations of Food Addiction in a Sample Recruited to Be Nationally Representative of the United States. European Eating Disorders Review, 26(2), 112-119.

Schulte, E. M., Yokum, S., Potenza, M. N., \& Gearhardt, A. N. (2016). Neural systems implicated in obesity as an addictive disorder: from biological to behavioral mechanisms. In Progress in Brain Research (Vol. 223, pp. 329-346). Elsevier.

Sheehan, D. (1998). The Mini International Neuropsychiatric Interview (MINI): the development and validation of a structured diagnostic psychiatric interview. Journal of Clinical Psychiatry, 59(Suppl 20), 2233.

Siervo, M., Arnold, R., Wells, J. C. K., Tagliabue, A., Colantuoni, A., Albanese, E., ... Stephan, B. C. M. (2011). Intentional weight loss in overweight and obese individuals and cognitive function: a systematic review and meta-analysis. Obesity Reviews, 12(11), 968-983.

Smith, E., Hay, P., Campbell, L., \& Trollor, J. N. (2011). A review of the association between obesity and cognitive function across the lifespan: Implications for novel approaches to prevention and treatment. Obesity Reviews, 12(9), 740-755.

Smith, J. L., Mattick, R. P., Jamadar, S. D., \& Iredale, J. M. (2014). Deficits in behavioural inhibition in substance abuse and addiction: A meta-analysis. Drug and Alcohol Dependence, 145,1-33.

Song, T. F., Chi, L., Chu, C. H., Chen, F. T., Zhou, C., \& Chang, Y. K. (2016). Obesity, cardiovascular fitness, and inhibition function: An electrophysiological study. Frontiers in Psychology, 7:1124.

Stinson, E. J., Krakoff, J., \& Gluck, M. E. (2018). Depressive symptoms and poorer performance on the Stroop Task are associated with weight gain. Physiology and Behavior, 186, 25-30.

Treasure, J., Leslie, M., Chami, R., \& Fernández-Aranda, F. (2018). Are trans diagnostic models of eating 
Obesity, Food Addiction and Executive Functions -23disorders fit for purpose? A consideration of the evidence for food addiction. European Eating Disorders Review, 26(2), 83-91.

Tsai, C.-L., Huang, T.-H., \& Tsai, M.-C. (2017). Neurocognitive performances of visuospatial attention and the correlations with metabolic and inflammatory biomarkers in adults with obesity. Experimental Physiology, 102(12), 1683-1699.

Verdejo-García, A., Lozano, Ó., Moya, M., Alcázar, M. Á., \& Pérez-García, M. (2010). Psychometric properties of a Spanish version of the UPPS-P impulsive behavior scale: Reliability, validity and association with trait and cognitive impulsivity. Journal of Personality Assessment, 92(1), 70-77.

Veronese, N., Facchini, S., Stubbs, B., Luchini, C., Solmi, M., Manzato, E., ... Fontana, L. (2017). Weight loss is associated with improvements in cognitive function among overweight and obese people: A systematic review and meta-analysis. Neuroscience \& Biobehavioral Reviews, 72, 87-94.

Whiteside, S. P., Lynam, D. R., Miller, J. D., \& Reynolds, S. K. (2001). Validation of the UPPS Impulsive Behavior Scale: A four factor model of impulsivity. European Journal of Personality, 19(7), 559-574.

Wilson, G. T. (2010). Eating disorders, obesity and addiction. European Eating Disorders Review, 18(5), 341-351.

Wolz, I., Granero, R., \& Fernández-Aranda, F. (2017). A comprehensive model of food addiction in patients with binge-eating symptomatology: The essential role of negative urgency. Comprehensive Psychiatry, 74, 118-124.

Wolz, I., Hilker, I., Granero, R., Jiménez-Murcia, S., Gearhardt, A. N., Dieguez, C., ... FernándezAranda, F. (2016). "Food Addiction" in Patients with Eating Disorders is Associated with Negative Urgency and Difficulties to Focus on Long-Term Goals. Frontiers in Psychology, 7, 61. 
Obesity, Food Addiction and Executive Functions -24-

Yang, Y., Shields, G. S., Guo, C., \& Liu, Y. (2018). Executive function performance in obesity and overweight individuals: A meta-analysis and review. Neuroscience and Biobehavioral Reviews, 84, 225-244.

Ziauddeen, H., Alonso-Alonso, M., Hill, J. O., Kelley, M., \& Khan, N. A. (2015). Obesity and the Neurocognitive Basis of Food Reward and the Control of Intake. Advances in Nutrition, 6(4), 474486.

Table 1. Prevalence and severity level of food addiction measures and comparison between groups

\begin{tabular}{|c|c|c|c|c|c|c|c|c|c|}
\hline & & \multicolumn{2}{|c|}{ Control $(n=36)$} & \multicolumn{2}{|c|}{ Obesity $(n=33)$} & \multirow[b]{2}{*}{$\chi^{2}$} & \multirow[b]{2}{*}{$d f$} & \multirow[b]{2}{*}{$p$} & \\
\hline & & $n$ & $\%$ & $n$ & $\%$ & & & & \\
\hline \multirow[t]{2}{*}{ Food addiction } & Present & 1 & $2.8 \%$ & 8 & $24.2 \%$ & 6.99 & 1 & $.008 *$ & $0.66^{\top}$ \\
\hline & Absent & 35 & $97.2 \%$ & 25 & $75.8 \%$ & & & & \\
\hline Food addiction & Absent & 35 & $97.2 \%$ & 25 & $75.8 \%$ & 7.22 & 2 & $.027^{*}$ & $0.66^{\top}$ \\
\hline \multirow[t]{2}{*}{ severity } & Moderate & 0 & $0.0 \%$ & 3 & $9.1 \%$ & & & & $0.52^{\top}$ \\
\hline & Severe & 1 & $2.8 \%$ & 5 & $15.2 \%$ & & & & 0.44 \\
\hline \multicolumn{2}{|c|}{ Food addiction dimensional score } & Mean & $S D$ & Mean & $S D$ & $F$ & $d f$ & $p$ & \\
\hline \multicolumn{2}{|c|}{ Total DSM-5 food addiction criteria } & 0.67 & 1.47 & 2.64 & 2.86 & 13.26 & $1 ; 67$ & $.001^{*}$ & $\mathbf{0 . 8 7 ^ { \top }}$ \\
\hline
\end{tabular}

Note. SD: standard deviation. df: degrees of freedom. *Bold: significant comparison (.05 level).

${ }^{\dagger}$ Effect size in the moderate $(|d|>0.50)$ to high range $(|d|>0.80)$. 
Obesity, Food Addiction and Executive Functions -25Table 2. Association between food addiction severity (total number of criteria) with neuropsychological scores, impulsivity and eating disorder severity: partial correlation coefficients

\begin{tabular}{|c|c|c|}
\hline & $\begin{array}{l}\text { Controls } \\
(n=36)\end{array}$ & $\begin{array}{l}\text { Obesity } \\
(n=33)\end{array}$ \\
\hline \multicolumn{3}{|l|}{${ }^{1}$ Neuropsychological scores } \\
\hline IGT: Total score & -.032 & $-.267^{\dagger}$ \\
\hline IGT: Block 1 & -.084 & $-.248^{\dagger}$ \\
\hline IGT: Block 2 & -.042 & .025 \\
\hline IGT: Block 3 & -.078 & $-.285^{\top}$ \\
\hline IGT: Block 4 & -.010 & -.238 \\
\hline IGT: Block 5 & .072 & -.185 \\
\hline CPT: Omissions & .132 & .163 \\
\hline CPT: Commissions & .078 & .092 \\
\hline CPT: Reaction time & -.052 & .104 \\
\hline CPT: Detectability & -.134 & -.180 \\
\hline CPT: Perseverations & .135 & .167 \\
\hline \multicolumn{3}{|l|}{${ }^{2}$ Impulsivity } \\
\hline UPPS-P: Lack of premeditation & $.305^{\top}$ & -.033 \\
\hline UPPS-P: Lack of perseverance & $.493^{\top}$ & .005 \\
\hline UPPS-P: Sensation seeking & .005 & .200 \\
\hline UPPS-P: Positive urgency & $.447^{\dagger}$ & .092 \\
\hline UPPS-P: Negative urgency & $.405^{\dagger}$ & $.492^{\dagger}$ \\
\hline \multicolumn{3}{|l|}{${ }^{2} E D$ severity } \\
\hline EDI-2: Total & $.445^{\top}$ & .147 \\
\hline
\end{tabular}

Note. IGT: lowa Gambling Task. CPT: Conners' Continuous Performance Test. ED: eating disorder. EDI-2: Eating Disorders Inventory-2

${ }^{1}$ Results adjusted for age and years of schooling. ${ }^{2}$ Results adjusted for age.

${ }^{\dagger}$ Effect size in the moderate $(|r|>0.24)$ to high range $(|r|>0.37)$. 
Obesity, Food Addiction and Executive Functions -26-

Table 3. Comparison between food addiction groups: ANOVA

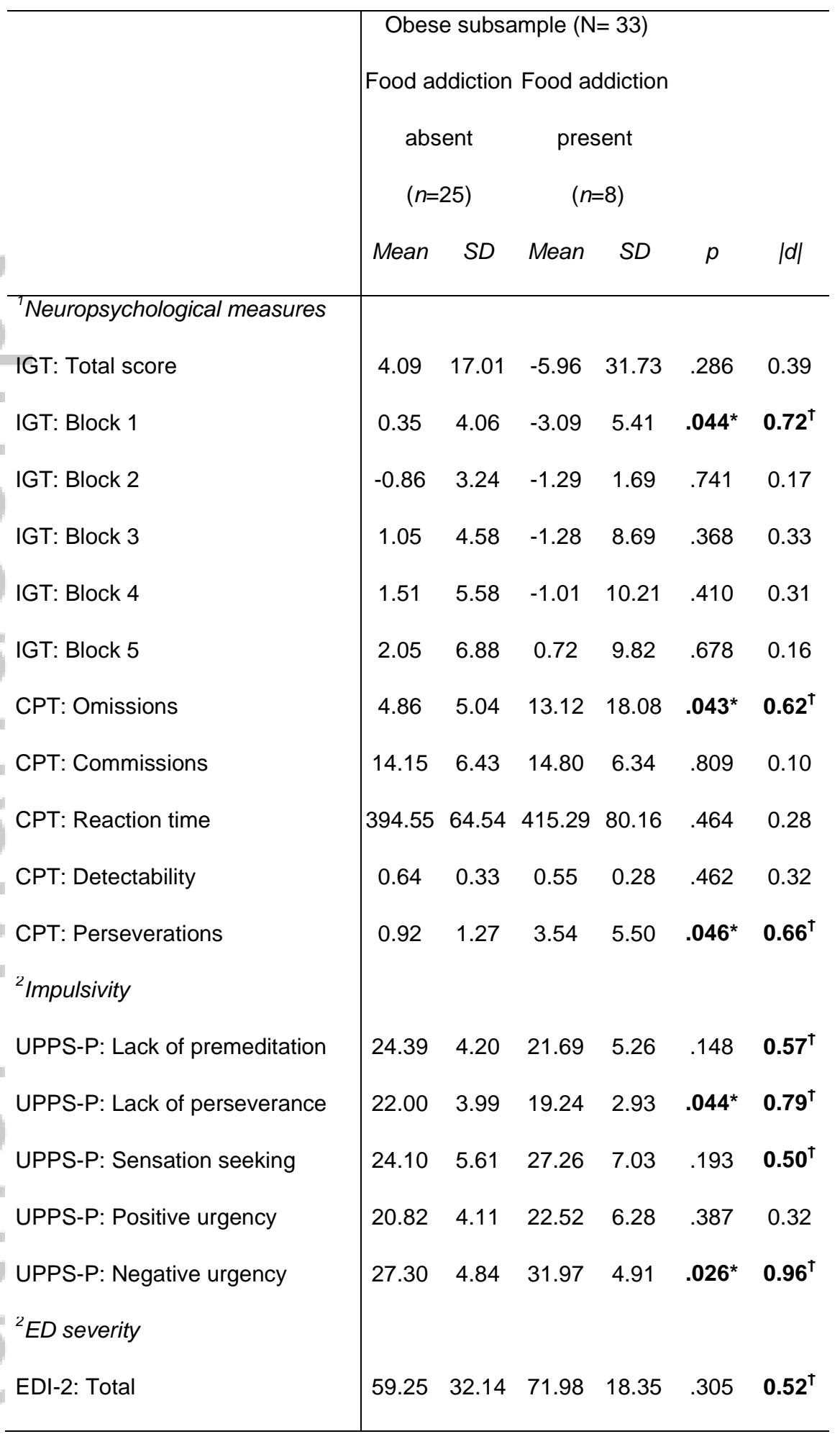

Note. IGT: Iowa Gambling Task. CPT: Conners' Continuous Performance Test. ED: eating disorders. EDI-2: Eating Disorders Inventory-2 
Obesity, Food Addiction and Executive Functions -27-

${ }^{1}$ ANOVA adjusted for age and years of school attendance. ${ }^{2}$ ANOVA adjusted for age.

*Bold: significant comparison (.05 level). ${ }^{\dagger}$ Effect size into the moderate $(|d|>0.50)$ to high range $(|d|>0.80)$.

This article is protected by copyright. All rights reserved. 
Obesity, Food Addiction and Executive Functions -28-

Figure 1. Prevalence and severity level of food addiction between groups. FA: Food addiction.

This article is protected by copyright. All rights reserved. 
Obesity, Food Addiction and Executive Functions -29-

Figure 2. First panel: IGT total scores for OB patients $(n=33)$ meeting and not meeting food addiction criteria (results adjusted for age and years of school attendance). Second panel: scatterplot for the distribution of the IGT total score and total food addiction criteria for OB patients. FA: food addiction.

This article is protected by copyright. All rights reserved. 


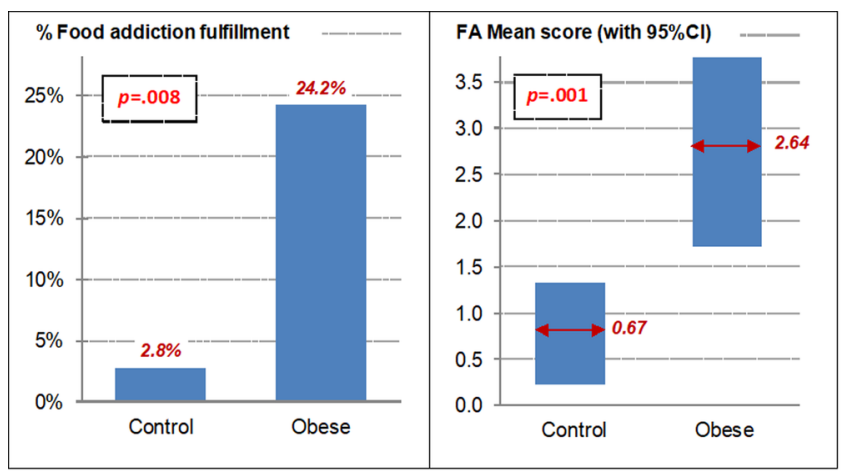

ERV_2636_F1.tif

This article is protected by copyright. All rights reserved. 


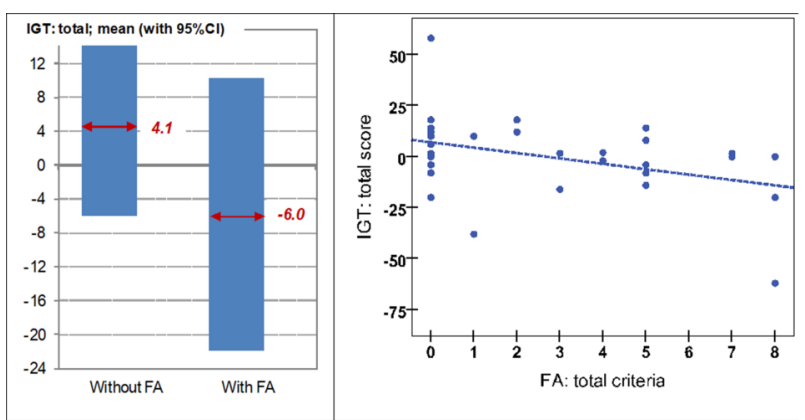

ERV_2636_F2.tif

This article is protected by copyright. All rights reserved. 


\section{University Library}

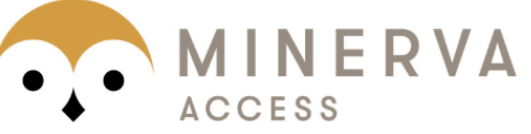

A gateway to Melbourne's research publications

Minerva Access is the Institutional Repository of The University of Melbourne

\section{Author/s:}

Steward, T;Mestre-Bach, G;Vintro-Alcaraz, C;Lozano-Madrid, M;Aguera, Z;FernandezFormoso, JA;Granero, R;Jimenez-Murcia, S;Vilarrasa, N;Garcia-Ruiz-de-Gordejuela, A;Veciana de las Heras, M;Custal, N;Virgili, N;Lopez-Urdiales, R;Gearhardt, AN;Menchon, JM;Soriano-Mas, C;Fernandez-Aranda, F

Title:

Food addiction and impaired executive functions in women with obesity

Date:

2018-11-01

\section{Citation:}

Steward, T., Mestre-Bach, G., Vintro-Alcaraz, C., Lozano-Madrid, M., Aguera, Z., FernandezFormoso, J. A., Granero, R., Jimenez-Murcia, S., Vilarrasa, N., Garcia-Ruiz-de-Gordejuela, A., Veciana de las Heras, M., Custal, N., Virgili, N., Lopez-Urdiales, R., Gearhardt, A. N., Menchon, J. M., Soriano-Mas, C. \& Fernandez-Aranda, F. (2018). Food addiction and impaired executive functions in women with obesity. EUROPEAN EATING DISORDERS REVIEW, 26 (6), pp.574-584. https://doi.org/10.1002/erv.2636.

Persistent Link:

http://hdl.handle.net/11343/285037 